\title{
Factors Associated with Multiple Hospital Readmissions for Individuals with Spinal Cord Injury
}

\author{
ALEXANDRA CANORI, DPT'; AMIT KUMAR, MPH, PHD ${ }^{2,3}$; SHIVAYOGI V. HIREMATH, \\ $\mathrm{PHD}^{1}$ \\ ${ }^{1}$ Department of Health and Rehabilitation Sciences, College of Public Health, Temple University \\ ${ }^{2}$ Center for Health Equity Research, Northern Arizona University \\ ${ }^{3}$ Department of Physical Therapy and Athletic Training, Northern Arizona University \\ Correspondence: $\underline{\text { Shiv.Hiremath@temple.edu (Shivayogi V. Hiremath) }}$
}

\begin{abstract}
Objective: To identify patient-level and clinical factors associated with multiple hospital readmissions in individuals with spinal cord injury (SCI). Design: Retrospective cohort analysis of the publicly available dataset from the SCI Rehabilitation (SCIRehab) study. Setting: Six rehabilitation centers in the U.S. that participated in the SCIRehab study. Participants: Individuals with traumatic SCI $(\mathrm{N}=1371)$ who were consecutively enrolled in the SCIRehab study. Outcome measures: The primary outcome was all-cause hospital readmission within 1 year of discharge from a rehabilitation center. The patient-level and clinical factors include employment status, depression, caregiver support, state-funded insurance, functional status, and rehabilitation services. Results: Of the 1170 participants included in the study, 228 were readmitted once and 120 were readmitted multiple times. In our study, 34.2\% and 10.8\% were readmitted more than once due to genitourinary and respiratory conditions, respectively. Lower utilization of rehabilitation services, lower functional status, unemployment, and depression were associated with higher odds of being readmitted multiple times within one year of discharge from inpatient rehabilitation facility. Conclusion: Psychosocial and clinical factors were associated with increased risk. for multiple readmissions in individuals with SCI. In order to reduce recurrent readmissions in individuals with SCI, further research is necessary to maximize efficacy of risk. factor modification and prevention strategies.
\end{abstract}

\section{Introduction}

Hospital readmissions are a pervasive manifestation throughout health care systems that can carry detrimental repercussions on patient recovery. ${ }^{1}$ Readmissions rates within 30 days of discharge from inpatient rehabilitation facility (IRF) has ranged from 5.8\%-18.8\%. ${ }^{2}$ Due to rising costs of health care as sociated with hospital readmis sions in the United States, the Center for Medicare and Medicaid Services (CMS) has implemented the IRF quality reporting requirements and the Hospital Readmission Reduction Program (HRRP) to improve quality of care. The IRF quality reporting requirements necessitate that IRFs report changes in skin integrity (pressure ulcers/injury), catheter-associated urinary tract infections, and functional status of Medicare patients. ${ }^{3}$ As a part of the HRRP and IRF quality reporting program on "All-Cause Unplanned Readmissions," hospitals and IRFs will receive penalties in reimbursement for their services if their readmission rates are above the national average rate. ${ }^{4,5}$ In order to avoid financial 
consequence, hospitals and IRFs have established guidelines and procedures to prevent hospital readmissions, which have reduced readmission rates for targeted conditions from $21.5 \%$ to $17.8 \% .4,6$ As readmissions declined due to the HRRP, Medicare costs declined $\$ 2.28$ billion compared to the cost of readmissions in $2010 .^{7}$ The CMS stipulates that hospital readmissions are potentially preventable if they can be avoided by: adequate prophylaxis, provision of quality care during initial hospitalization, adequate dis charge planning and post-discharge follow-up, or improved communication between health care teams with clearly mandated delineation of responsibilities.-10 However, potentially preventable readmissions have not been defined specifically for individuals with spinal cord injury (SCI).

Hospital readmissions are especially prevalent in individuals with SCI. Despite efforts to reduce hospital readmissions, previous studies on health care utilization have shown that rates for hospital readmission following traumatic SCI vary from $36.2 \%$ to as high as $57.3 \%$ in the first 12 months following the injury. 11 ${ }_{13}$ Furthermore, $12.5 \%$ of individuals who sustained new SCI were readmitted at least twice within one year following injury. ${ }^{13}$ DeJong et al. found that individuals with cervical level American Spinal Injury Association impairment scale (AIS) grade A-C injuries have the highest average number of readmissions and length of stay, followed by all individuals with AIS grade D, and paraplegia grades A-C have the lowest average number of readmissions and length of stay. ${ }^{13}$ The most prevalent causes for hospital readmission among patients with SCI are health conditions related to the genitourinary system, respiratory system, and skin and subcutaneous tissues. ${ }^{13,14}$ Improved management of genitourinary and skin conditions for individuals with SCI could result in substantial reduction in morbidity and cost savings..$^{15}$ Studies on health care utilization following SCI have shown that discharge functional status, comorbidities, previous hospitalizations, enrollment in Medicare or Medicaid as their primary insurance provider, and length of stay at IRF were independent contributors to readmission rates. ${ }^{14,16-18}$

Efforts to improve the continuum of care to reduce readmissions after SCI have been multidimensional. However, Stillman et al. ${ }^{19}$ identified barriers for patients with SCI in $76.9 \%$ of primary care offices that participated in their study. Caregiver support and caregiver availability for patients with SCI after discharge from IRF may influence readmission rates as well. ${ }^{5}$ DeJong et al. ${ }^{13}$ found that the number of readmissions varies across facilities, which is likely representing discrepancies in access to outpatient care depending on location. Therefore, inaccessibility of outpatient care may also contribute to increased rate of readmission. While factors that frequently lead to single hospital readmission after SCI have been recognized, risk factors of multiple hospital readmissions related to post-acute rehabilitation practice, discharge planning strategies and caregiver support, and psychosocial support remain unknown and create a crucial knowledge gap that this study hopes to fill.

Previous research has discussed AIS impairment level, length of stay, and rehabilitation center in relation to multiple readmissions, ${ }^{13}$ but the contribution of psychosocial risk factors and rehabilitation intensity to multiple readmissions following discharge from IRF has not been explored in individuals with SCI. This is primarily due to a lack of data that portrays utilization of rehabilitation services, health outcomes, and social risk factors. This study aimed to provide new understanding of multiple hospital readmissions that may assist clinicians and health care institutions in delivering appropriate post-acute care for patients with SCI to minimize the reoccurrence of preventable hospitalizations. The primary objective of this study was to expand upon the work of DeJong et allis surrounding multiple readmissions in individuals with SCI by examining psychosocial and clinical factors associated with multiple readmissions after discharge from an IRF. A secondary objective of this study was to evaluate the effect of genitourinary, respiratory, and skin conditions on multiple readmissions in this population, because these conditions are preventable and common contributors to single readmissions. ${ }^{13,14}$ Based on prior research, $, 6,8,10,12-21$ we hypothesized that psychosocial risk factors and lower utilization of rehabilitation services would be associated with multiple readmissions within one year of discharge from an IRF. 


\section{Methods}

\section{Data Source}

We conducted a retrospective secondary analysis of the publicly available dataset from the SCIRehab project (https://www.icpsr.umich.edu/icpsrweb/ADDEP/studies/36724). The team conducting the SCIRehab project obtained informed consent prior to enrolling eligible participants, and each rehabilitation center that participated in the study obtained institutional review board (IRB) approval. The SCIRehab project collected data from 1,376 individuals with SCI who received treatment from six nonprofit, geographically dispersed rehabilitation facilities in the United States between 2007 and 2009, and patient interviews were conducted at six months and one year following IRF discharge. ${ }^{21}$ The SCIRehab project sought to develop specific guidelines for acute rehabilitation of SCI by collecting thorough patient history and detailed documentation of treatment sessions from seven disciplines. The SCIRehab project inves tigators then studied the content of these treatment sessions and accompanying patient outcomes. ${ }^{21,22}$ Detailed information regarding the SCIRehab project is available at the Interuniversity Consortium for Political and Social Research (ICPSR) archive (https://www.icpsr.umich.edu/icpsrweb/ADDEP/studies/36724). For this study, we agreed to the ICPSR's data use policies and accessed the publicly available data from the ICPSR archive. Our university's IRB reviewed our protocol and indicated that because the data is de-identified and publicly available, the analysis of this data does not require an IRB approval.

\section{Cohort Selection}

The population of interest was individuals who were part of the SCIRehab project $(n=1376)$. The SCIRehab project included individuals who were 12 years old or older, diagnosed with traumatic SCI, and participated in the study's 12-month post-injury follow-up survey. The final cohort of study participants (Figure 1) were subjects with information provided regarding occurrence of readmissions $(\mathrm{N}=1170)$. We have excluded 206 individuals from the denominator (study cohort) due to missing information on readmissions, as we were unable to determine their analysis group.

Figure 1. Process of cohort selection from all data and number of participants with multiple readmissions for genitourinary, respiratory, and skin related health complications. Individuals are further subdivided by whether they were readmitted once or more than once in the course of the year for each condition.

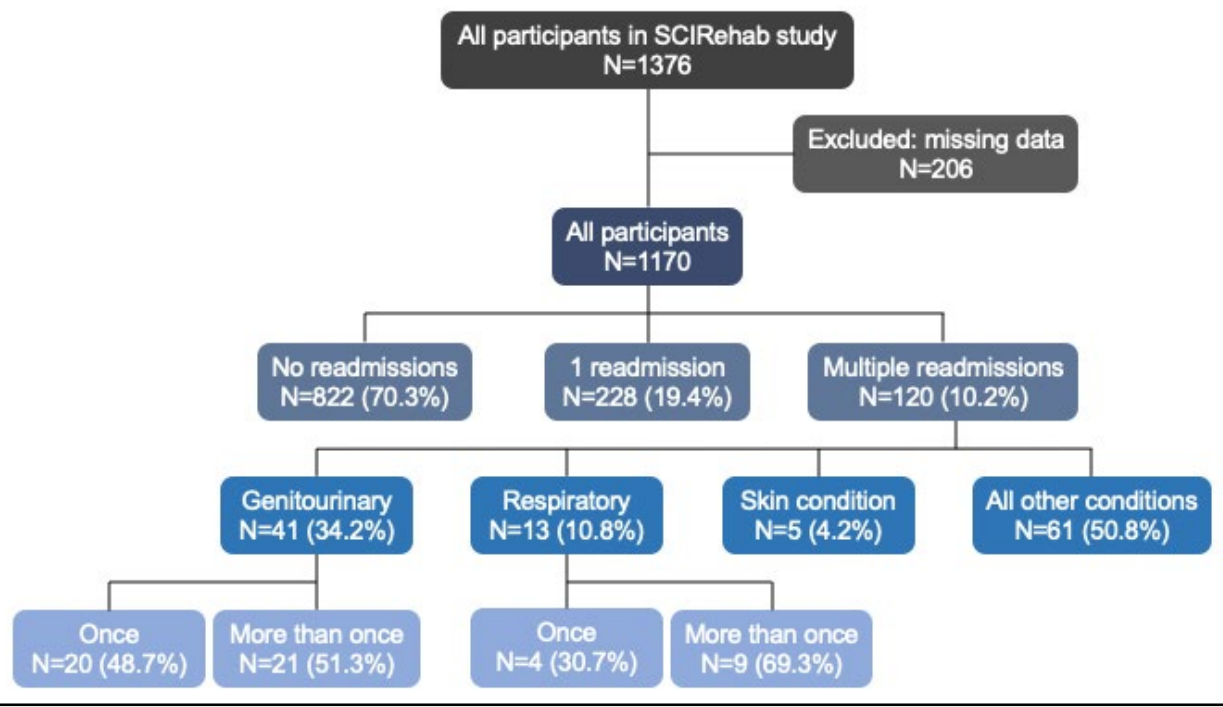




\section{Study Outcome}

The primary outcome was number of hospital readmissions (none, one, or multiple) between the time of discharge from an IRF and the one-year follow-up date. We identified causes of readmissions and analyzed how the patients' demographic, clinical, and psychosocial factors were associated with hospital readmission stratified by three groups (no readmissions, one readmission, and multiple readmissions). All groups of readmissions were mutually exclusive.

\section{Study Covariates}

The study covariates were derived during the study design phase from previous literature and post-acute rehabilitation quality framework. ${ }^{23}$ We have included many patient-level and clinical variables to control for case-mix severity of injury, including the American Spinal Injury Association (ASIA) Motor Index Score, use of mechanical ventilation, mode of locomotion, and type of wheelchair. The motor component of Functional Independence Measure (FIM) is reliable and valid, 24, 25 and was used to determine level of independence with functional mobility at admission to IRF, discharge from IRF, and one-year post discharge follow-up from IRF. We as sessed tolerance and Variance Inflation Factor (VIF) and found no collinearity between the covariates with respect to readmissions.

\section{Demographic and Psychosocial Factors}

The following demographic and psychosocial factors were assessed: age, sex, race, ethnicity, education, occupation, and marital status. Patient survey ratings from the SCI Model System Form I and Form II along with 6- and 12-month post-injury interviews ${ }^{21}$ were used to evaluate each of the following psychosocial factors: living arrangements, private place of residence, accessibility of residence, access to transportation, primary caregiver, whether primary caregiver received training during IRF stay, primary insurance type, number of hours of paid assistance per day, number of hours of unpaid assistance per day, and community participation. Depression was assessed using the Personal Health Questionnaire-9, which is a valid, 9-item measure of self-reported depressive symptoms. ${ }^{26}$

\section{Rehabilitation Services and Clinical Factors}

Total hours in each of the following disciplines were analyzed: physical therapy, occupational therapy, speech-language pathology, therapeutic recreation, nursing, psychology, and social work. This information was originally obtained for the SCIRehab study by chart abstraction. ${ }^{21}$ Total hours of nursing only includes treatments. Care management and patient education were documented separately because information in the chart was deemed insufficient. ${ }^{21}$ The following clinical factors were also examined: traumatic etiology, spinal surgery, associated injuries, number of days in IRF, number of days from injury to initial IRF admission, total readmissions within one year of discharge from IRF, and hours of therapy per day at IRF.

\section{Statistical Analyses}

We divided the participants into three groups, based on number of readmissions: individuals who were not readmitted, readmitted once, and readmitted multiple times (greater than or equal to two). Descriptive statistics were used to describe the study cohort by three groups of readmission. The KolmogorovSmirnov normality test was completed, and since no data was normally distributed, we reported median (range) values in addition to mean (SD) values for all continuous variables presented in Table 1 . In addition, chi-square and one-way analysis of variance (ANOVA) analyses were performed for categorical 
and continuous variables, respectively. Multivariate logistic regression was conducted to determine the association of patient demographics, clinical, and psychosocial characteristics with number of readmissions. Odds Ratios (OR) with a 95\% Confidence Interval (CI) were reported for the three different groups. Prevalence of hospital readmissions was cross examined with 38 demographic, psychosocial, and clinical factors to obtain the mean value and standard deviation (Table 1). Logistic regression OR was calculated for all variables, and adjusted OR was performed for significant variables which are presented in Table 2. All statistical analyses were performed using SAS 9.4 software (SAS Institute, Cary, NC).

Table 1. Patient demographics, clinical information, and psychosocial factors by three groups of readmission. Values are reported as frequency (\%) or mean \pm standard deviation and median (minimum-maximum).

\begin{tabular}{|c|c|c|c|}
\hline Variable & $\begin{array}{l}\text { No } \\
\text { Readmissions } \\
\text { N=822 }(70.2 \%)\end{array}$ & $\begin{array}{l}\text { One } \\
\text { Readmission } \\
\mathrm{N}=228(19.4 \%)\end{array}$ & $\begin{array}{l}\text { Multiple } \\
\text { Readmissions } \\
\mathrm{N}=120(10.2 \%)\end{array}$ \\
\hline \multicolumn{4}{|l|}{ Demographics } \\
\hline \multicolumn{4}{|l|}{ Age } \\
\hline 12-19 & $131(11.2 \%)$ & $23(2.0 \%)$ & $24(2.1 \%)$ \\
\hline $20-29$ & $240(20.5 \%)$ & $48(4.1 \%)$ & $30(2.6 \%)$ \\
\hline $30-39$ & $124(10.6 \%)$ & $44(3.8 \%)$ & $16(1.4 \%)$ \\
\hline $40-49$ & $130(11.1 \%)$ & $48(4.1 \%)$ & $14(1.2 \%)$ \\
\hline $50-59$ & $111(9.5 \%)$ & $30(2.6 \%)$ & $19(1.6 \%)$ \\
\hline $60-69$ & $62(5.3 \%)$ & $25(2.1 \%)$ & $12(1.0 \%)$ \\
\hline \multirow[t]{2}{*}{70 and older } & $24(2.1 \%)$ & $10(0.9 \%)$ & $5(0.4 \%)$ \\
\hline & $\mathrm{n}=822$ & $\mathrm{n}=228$ & $\mathrm{n}=120$ \\
\hline \multicolumn{4}{|l|}{ Sex } \\
\hline Male & $667(57.0 \%)$ & $179(15.3 \%)$ & $100(8.6 \%)$ \\
\hline \multirow{2}{*}{ Female } & $155(13.3 \%)$ & $49(4.2 \%)$ & $20(1.7 \%)$ \\
\hline & $\mathrm{n}=822$ & $\mathrm{n}=228$ & $\mathrm{n}=120$ \\
\hline \multicolumn{4}{|l|}{ Race } \\
\hline Caucasian & $604(51.6 \%)$ & $166(14.2 \%)$ & $75(6.4 \%)$ \\
\hline African American & $157(13.4 \%)$ & $51(4.4 \%)$ & $34(2.9 \%)$ \\
\hline \multirow[t]{2}{*}{ Asian/pacific islander } & $17(1.5 \%)$ & $5(0.4 \%)$ & $3(0.3 \%)$ \\
\hline & $\mathrm{n}=778$ & $\mathrm{n}=222$ & $\mathrm{n}=112$ \\
\hline \multicolumn{4}{|l|}{ Ethnicity } \\
\hline Not Hispanic & $747(63.9 \%)$ & $209(17.9 \%)$ & $107(9.2 \%)$ \\
\hline \multirow[t]{2}{*}{ Hispanic } & $66(5.6 \%)$ & $19(1.6 \%)$ & $13(1.1 \%)$ \\
\hline & $\mathrm{n}=813$ & $\mathrm{n}=228$ & $\mathrm{n}=120$ \\
\hline \multicolumn{4}{|l|}{ Education* } \\
\hline $11^{\text {th }}$ grade or less & $160(13.7 \%)$ & $34(2.9 \%)$ & $33(2.8 \%)$ \\
\hline \multirow[t]{2}{*}{ High school or advanced degree } & $610(52.1 \%)$ & $174(14.9 \%)$ & $71(6.1 \%)$ \\
\hline & $\mathrm{n}=770$ & $\mathrm{n}=208$ & $\mathrm{n}=104$ \\
\hline \multicolumn{4}{|l|}{ Occupation* } \\
\hline Working/ home-maker & $548(46.8 \%)$ & $164(14.2 \%)$ & $65(5.6 \%)$ \\
\hline Unemployed/ retired & $113(9.7 \%)$ & $40(3.4 \%)$ & $27(2.3 \%)$ \\
\hline \multirow[t]{2}{*}{ Student } & $147(12.6 \%)$ & $20(1.7 \%)$ & $20(1.7 \%)$ \\
\hline & $\mathrm{n}=808$ & $\mathrm{n}=224$ & $\mathrm{n}=112$ \\
\hline
\end{tabular}




\begin{tabular}{|c|c|c|c|}
\hline Variable & $\begin{array}{l}\text { No } \\
\text { Readmissions } \\
\mathrm{N}=822(70.2 \%)\end{array}$ & $\begin{array}{l}\text { One } \\
\text { Readmission } \\
\mathrm{N}=228(19.4 \%)\end{array}$ & $\begin{array}{l}\text { Multiple } \\
\text { Readmissions } \\
\mathrm{N}=120(10.2 \%)\end{array}$ \\
\hline \multicolumn{4}{|l|}{ Severity of Injury } \\
\hline \multicolumn{4}{|l|}{ ASIA Motor Index Score* } \\
\hline C1-C4 ASIA A, B, C & $75(19.7 \%)$ & $29(31.2 \%)$ & $20(35.7 \%)$ \\
\hline C5-C8 ASIA A, B, C & $91(23.9 \%)$ & $15(16.1 \%)$ & $10(17.9 \%)$ \\
\hline T1-S5 ASIA A, B, C & $134(35.2 \%)$ & $32(34.3 \%)$ & $21(37.5 \%)$ \\
\hline \multirow[t]{2}{*}{ All ASIA D } & $81(21.3 \%)$ & $17(18.3 \%)$ & $5(8.9 \%)$ \\
\hline & $\mathrm{n}=381$ & $\mathrm{n}=93$ & $\mathrm{n}=56$ \\
\hline \multicolumn{4}{|l|}{ Mechanical ventilation at IRF* } \\
\hline Used & $101(8.6 \%)$ & $51(4.4 \%)$ & $20(1.7 \%)$ \\
\hline \multirow[t]{2}{*}{ Not used } & $717(61.3 \%)$ & $177(15.1 \%)$ & $99(8.5 \%)$ \\
\hline & $\mathrm{n}=818$ & $\mathrm{n}=228$ & $\mathrm{n}=119$ \\
\hline \multicolumn{4}{|l|}{ Mode of locomotion* } \\
\hline Walking & $144(12.3 \%)$ & $20(1.7 \%)$ & $7(0.6 \%)$ \\
\hline \multirow[t]{2}{*}{ Wheelchair } & $677(57.9 \%)$ & $207(17.7 \%)$ & $113(9.7 \%)$ \\
\hline & $\mathrm{n}=821$ & $\mathrm{n}=227$ & $\mathrm{n}=120$ \\
\hline \multicolumn{4}{|l|}{ Type of wheelchair* } \\
\hline Manual wheelchair & $362(30.9 \%)$ & $83(7.1 \%)$ & $51(4.4 \%)$ \\
\hline Power assist wheelchair/ scooter & $13(1.1 \%)$ & $2(0.2 \%)$ & $1(0.1 \%)$ \\
\hline Power wheelchair & $195(16.7 \%)$ & $99(8.5 \%)$ & $54(4.6 \%)$ \\
\hline \multirow[t]{2}{*}{ N/A- no wheelchair use } & $249(21.3 \%)$ & $42(3.6 \%)$ & $14(1.2 \%)$ \\
\hline & $\mathrm{n}=819$ & $\mathrm{n}=226$ & $\mathrm{n}=120$ \\
\hline \multicolumn{4}{|l|}{ Clinical Information } \\
\hline \multirow[t]{3}{*}{ FIM Motor at admission* } & $24.4 \pm 11.3$ & $22.1 \pm 10.9$ & $21.4 \pm 10.0$ \\
\hline & $22.0(13.0-82.0)$ & $18.0(13.0-62.0)$ & $17.0(13.0-56.0)$ \\
\hline & $\mathrm{n}=822$ & $\mathrm{n}=228$ & $\mathrm{n}=120$ \\
\hline \multirow[t]{3}{*}{ FIM Motor at discharge* } & $55.0 \pm 21.6$ & $45.9 \pm 22.8$ & $42.1 \pm 21.8$ \\
\hline & $63.0(13.0-89.0)$ & $40.0(13.0-88.0)$ & $38.0(13.0-80.0)$ \\
\hline & $\mathrm{n}=822$ & $\mathrm{n}=228$ & $\mathrm{n}=120$ \\
\hline \multirow[t]{3}{*}{ FIM Motor 1 year follow-up* } & $66.7 \pm 25.2$ & $52.0 \pm 27.2$ & $48.1 \pm 26.7$ \\
\hline & $79.0(13.0-91.0)$ & $53.0(13.0-91.0)$ & $45.0(13.0-91.0)$ \\
\hline & $\mathrm{n}=782$ & $\mathrm{n}=217$ & $\mathrm{n}=111$ \\
\hline \multicolumn{4}{|l|}{ Traumatic etiology* } \\
\hline Vehicular & $412(35.2 \%)$ & $117(10.0 \%)$ & $69(5.9 \%)$ \\
\hline Sports & $103(8.8 \%)$ & $22(1.9 \%)$ & $10(0.6 \%)$ \\
\hline Violence & $81(6.9 \%)$ & $19(1.6 \%)$ & $15(1.3 \%)$ \\
\hline Fall & $191(16.3 \%)$ & $62(5.3 \%)$ & $22(1.9 \%)$ \\
\hline \multirow{2}{*}{ Medical/ other } & $35(2.1 \%)$ & $8(0.7 \%)$ & $4(0.3 \%)$ \\
\hline & $\mathrm{n}=822$ & $\mathrm{n}=228$ & $\mathrm{n}=120$ \\
\hline \multicolumn{4}{|l|}{ Spinal surgery } \\
\hline Yes & $679(58.2 \%)$ & $190(16.3 \%)$ & $95(8.1 \%)$ \\
\hline \multirow{2}{*}{ No } & $140(12.0 \%)$ & $38(3.2 \%)$ & $25(2.1 \%)$ \\
\hline & $\mathrm{n}=819$ & $\mathrm{n}=228$ & $\mathrm{n}=120$ \\
\hline \multicolumn{4}{|l|}{ Associated injuries } \\
\hline Yes & $333(28.5 \%)$ & $106(9.1 \%)$ & $63(5.4 \%)$ \\
\hline \multirow[t]{2}{*}{ No } & $487(41.7 \%)$ & $121(10.4 \%)$ & $57(4.9 \%)$ \\
\hline & $\mathrm{n}=820$ & $\mathrm{n}=227$ & $\mathrm{n}=120$ \\
\hline \multirow[t]{3}{*}{ Number of days in IRF } & $56.2 \pm 36.0$ & $58.7 \pm 37.2$ & $60.5 \pm 45.2$ \\
\hline & $46.0(2.0-325.0)$ & $49.0(2.0-254.0)$ & $45.0(9.0-248.0)$ \\
\hline & $\mathrm{n}=822$ & $\mathrm{n}=228$ & $\mathrm{n}=120$ \\
\hline
\end{tabular}




\begin{tabular}{|c|c|c|c|}
\hline Variable & $\begin{array}{l}\text { No } \\
\text { Readmissions } \\
\mathrm{N}=822(70.2 \%)\end{array}$ & $\begin{array}{l}\text { One } \\
\text { Readmission } \\
\mathrm{N}=228(19.4 \%)\end{array}$ & $\begin{array}{l}\text { Multiple } \\
\text { Readmissions } \\
\mathrm{N}=120(10.2 \%)\end{array}$ \\
\hline Number of days from injury to initial IRF admission* & $\begin{array}{l}27.7 \pm 25.4 \\
20.0(2.0-275.0) \\
n=822\end{array}$ & $\begin{array}{l}36.4 \pm 31.5 \\
27.0(3.0-219.0) \\
n=227\end{array}$ & $\begin{array}{l}36.9 \pm 31.8 \\
31.0(5.0-128.0) \\
n=120\end{array}$ \\
\hline Total readmissions within 1 year of discharge & $\begin{array}{l}0.0 \pm 0.0 \\
0.0(0.0-0.0) \\
\mathrm{n}=822\end{array}$ & $\begin{array}{l}1.0 \pm 0.0 \\
1.0(1.0-1.0) \\
\mathrm{n}=228\end{array}$ & $\begin{array}{l}2.7 \pm 1.3 \\
2.0(2.0-9.0) \\
n=120\end{array}$ \\
\hline Physical therapy total hours* & $\begin{array}{l}59.0 \pm 36.0 \\
48.1(1.5-187.2) \\
n=822\end{array}$ & $\begin{array}{l}54.3 \pm 34.7 \\
44.5(2.9-204.0) \\
n=228\end{array}$ & $\begin{array}{l}50.0 \pm 29.6 \\
44.4(6.2-142.7) \\
n=120\end{array}$ \\
\hline Occupational therapy total hours & $\begin{array}{l}53.7 \pm 36.8 \\
42.3(1.8-246.5) \\
n=822\end{array}$ & $\begin{array}{l}54.9 \pm 35.5 \\
44.5(6.0-197.9) \\
n=228\end{array}$ & $\begin{array}{l}51.8 \pm 32.9 \\
43.3(7.5-190.7) \\
n=120\end{array}$ \\
\hline Speech language pathology total hours* & $\begin{array}{l}3.7 \pm 9.1 \\
0.0(0.0-94.7) \\
n=822\end{array}$ & $\begin{array}{l}4.5 \pm 9.2 \\
0.0(0.0-59.2) \\
n=228\end{array}$ & $\begin{array}{l}8.0 \pm 20.5 \\
0.8(0.0-137.8) \\
n=120\end{array}$ \\
\hline Therapeutic recreation total hours* & $\begin{array}{l}19.8 \pm 17.5 \\
16.0(0.0-124.9) \\
n=822\end{array}$ & $\begin{array}{l}16.7 \pm 13.2 \\
15.1(0.0-82.9) \\
n=228\end{array}$ & $\begin{array}{l}15.7 \pm 11.9 \\
14.0(0.0-54.4) \\
n=120\end{array}$ \\
\hline Nursing total hours* & $\begin{array}{l}33.8 \pm 22.5 \\
29.7(1.5-225.4) \\
n=822\end{array}$ & $\begin{array}{l}38.7 \pm 24.0 \\
35.8(1.0-127.5) \\
n=228\end{array}$ & $\begin{array}{l}38.7 \pm 25.9 \\
33.2(3.8-156.6) \\
n=120\end{array}$ \\
\hline Psychology total hours & $\begin{array}{l}11.0 \pm 10.2 \\
8.0(0.0-73.3) \\
n=822\end{array}$ & $\begin{array}{l}10.9 \pm 10.0 \\
7.8(0.0-80.6) \\
\mathrm{n}=228\end{array}$ & $\begin{array}{l}11.3 \pm 10.9 \\
8.0(0.0-51.7) \\
n=120\end{array}$ \\
\hline Social work total hours & $\begin{array}{l}8.8 \pm 9.6 \\
5.8(0.04-73.8) \\
n=822\end{array}$ & $\begin{array}{l}9.4 \pm 10.2 \\
5.2(0.27-51.4) \\
n=228\end{array}$ & $\begin{array}{l}9.8 \pm 13.7 \\
5.4(0.0-78.4) \\
n=120\end{array}$ \\
\hline All disciplines total hours & $\begin{array}{l}189.7 \pm 105.2 \\
166.0(6.2-637.4) \\
n=822\end{array}$ & $\begin{array}{l}189.4 \pm 103.1 \\
171.7(15.8-639.5) \\
n=228\end{array}$ & $\begin{array}{l}185.3 \pm 109.0 \\
157.2(26.5-613.9) \\
n=120\end{array}$ \\
\hline Hours of therapy per day at IRF & $\begin{array}{l}3.6 \pm 0.7 \\
3.5(1.6-6.7) \\
n=822\end{array}$ & $\begin{array}{l}3.5 \pm 0.8 \\
3.4(1.4-6.5) \\
n=228\end{array}$ & $\begin{array}{l}3.5 \pm 0.8 \\
3.4(1.8-5.5) \\
n=120\end{array}$ \\
\hline Psychosocial Factors & & & \\
\hline $\begin{array}{l}\text { Depression* } \\
\text { Yes } \\
\text { No }\end{array}$ & $\begin{array}{l}44(4.1 \%) \\
711(66.3 \%) \\
n=755\end{array}$ & $\begin{array}{l}25(2.3 \%) \\
188(17.5 \%) \\
\mathrm{n}=213\end{array}$ & $\begin{array}{l}21(2.0 \%) \\
84(7.8 \%) \\
n=105\end{array}$ \\
\hline $\begin{array}{l}\text { Living arrangements } \\
\text { Lives alone } \\
\text { Lives with spouse/ significant other } \\
\text { Lives with unrelated roommate or attendant }\end{array}$ & $\begin{array}{l}430(36.8 \%) \\
336(28.7 \%) \\
26(2.2 \%) \\
n=792\end{array}$ & $\begin{array}{l}109(9.3 \%) \\
107(9.2 \%) \\
7(0.6 \%) \\
\mathrm{n}=223\end{array}$ & $\begin{array}{l}73(6.2 \%) \\
42(3.6 \%) \\
2(0.2 \%) \\
n=117\end{array}$ \\
\hline $\begin{array}{l}\text { Private place of residence } \\
\text { Yes } \\
\text { No }\end{array}$ & $\begin{array}{l}758(92.4 \%) \\
62(7.6 \%) \\
n=820\end{array}$ & $\begin{array}{l}197(86.4 \%) \\
31(13.6 \%) \\
n=228\end{array}$ & $\begin{array}{l}105(87.5 \%) \\
15(12.5 \%) \\
n=120\end{array}$ \\
\hline $\begin{array}{l}\text { Accessible residence: within home } \\
\text { Yes } \\
\text { No }\end{array}$ & $\begin{array}{l}760(67.0 \%) \\
41(3.6 \%) \\
n=801\end{array}$ & $\begin{array}{l}209(18.4 \%) \\
13(1.2 \%) \\
\mathrm{n}=222\end{array}$ & $\begin{array}{l}105(9.3 \%) \\
7(0.6 \%) \\
\mathrm{n}=112\end{array}$ \\
\hline
\end{tabular}




\begin{tabular}{|c|c|c|c|}
\hline Variable & $\begin{array}{l}\text { No } \\
\text { Readmissions } \\
\mathrm{N}=822(70.2 \%)\end{array}$ & $\begin{array}{l}\text { One } \\
\text { Readmission } \\
\mathrm{N}=228(19.4 \%)\end{array}$ & $\begin{array}{l}\text { Multiple } \\
\text { Readmissions } \\
\mathrm{N}=120(10.2 \%)\end{array}$ \\
\hline \multicolumn{4}{|l|}{ Accessible residence: enter/ exit* } \\
\hline Yes & $778(68.5 \%)$ & $210(18.5 \%)$ & $106(9.3 \%)$ \\
\hline \multirow[t]{2}{*}{ No } & $23(2.0 \%)$ & $13(1.1 \%)$ & $6(0.5 \%)$ \\
\hline & $\mathrm{n}=801$ & $\mathrm{n}=223$ & $\mathrm{n}=112$ \\
\hline \multicolumn{4}{|l|}{ Access to transportation* } \\
\hline Yes & $701(61.4 \%)$ & $182(16.0 \%)$ & $89(7.8 \%)$ \\
\hline \multirow[t]{2}{*}{ No } & $102(8.9 \%)$ & $42(3.7 \%)$ & $25(2.2 \%)$ \\
\hline & $\mathrm{n}=803$ & $\mathrm{n}=224$ & $\mathrm{n}=114$ \\
\hline \multicolumn{4}{|l|}{ Primary caregiver* } \\
\hline No one & $255(22.7 \%)$ & $41(3.6 \%)$ & $14(1.2 \%)$ \\
\hline Family/ significant other & $415(36.2 \%)$ & $134(11.7 \%)$ & $70(6.1 \%)$ \\
\hline \multirow{2}{*}{ Hired caregiver - private pay or state funded } & $121(10.6 \%)$ & $43(3.8 \%)$ & $28(2.5 \%)$ \\
\hline & $\mathrm{n}=791$ & $\mathrm{n}=218$ & $\mathrm{n}=112$ \\
\hline \multicolumn{4}{|l|}{ Caregiver trained at rehab* } \\
\hline Yes & $407(48.1 \%)$ & $121(14.3 \%)$ & $68(8.0 \%)$ \\
\hline \multirow[t]{2}{*}{ No } & $150(17.7 \%)$ & $66(7.8 \%)$ & $35(4.1 \%)$ \\
\hline & $\mathrm{n}=557$ & $\mathrm{n}=187$ & $\mathrm{n}=103$ \\
\hline \multicolumn{4}{|l|}{ Primary insurance type } \\
\hline Medicare & $49(4.0 \%)$ & $20(1.7 \%)$ & $12(1.0 \%)$ \\
\hline Medicaid & $136(11.6 \%)$ & $43(3.7 \%)$ & $25(2.1 \%)$ \\
\hline Worker's compensation & $83(7.1 \%)$ & $20(1.7 \%)$ & $14(1.2 \%)$ \\
\hline \multirow[t]{2}{*}{ Private } & $554(47.4 \%)$ & $145(12.4 \%)$ & $69(5.9 \%)$ \\
\hline & $\mathrm{n}=822$ & $\mathrm{n}=228$ & $\mathrm{n}=120$ \\
\hline \multirow[t]{3}{*}{ Number of hours paid assistance per day } & $2.9 \pm 6.5$ & $4.6 \pm 7.5$ & $6.4 \pm 8.8$ \\
\hline & $0.0(0.0-24.0)$ & $0.0(0.0-24.0)$ & $2.0(0.0-24.0)$ \\
\hline & $\mathrm{n}=794$ & $\mathrm{n}=222$ & $\mathrm{n}=117$ \\
\hline \multirow[t]{3}{*}{ Number of hours unpaid assistance per day } & $3.5 \pm 6.6$ & $5.5 \pm 8.2$ & $5.2 \pm 7.4$ \\
\hline & $0.0(0.0-24.0)$ & $1.0(0.0-24.0)$ & $2.0(0.0-24.0)$ \\
\hline & $\mathrm{n}=794$ & $\mathrm{n}=222$ & $\mathrm{n}=117$ \\
\hline \multicolumn{4}{|l|}{ Community participation* } \\
\hline Yes & $725(65.5 \%)$ & $188(17.0 \%)$ & $94(8.5 \%)$ \\
\hline \multirow[t]{2}{*}{ No } & $56(5.1 \%)$ & $29(2.6 \%)$ & $15(1.4 \%)$ \\
\hline & $\mathrm{n}=781$ & $\mathrm{n}=217$ & $\mathrm{n}=109$ \\
\hline
\end{tabular}

Chi square analysis was performed for categorical variables, and one-way ANOVA was performed for continuous variables. ${ }^{*} \mathrm{p}<0.05$ 
Table 2. Odds ratio (OR) analysis of significant patient demographics, severity of injury, psychosocial factors, and clinical information for individuals with one and multiple readmissions.

\begin{tabular}{|c|c|c|}
\hline Variable & $\begin{array}{l}\text { 1 Readmission } \\
\text { OR Point Estimate* (95\% CI) }\end{array}$ & $\begin{array}{l}\text { Multiple Readmissions } \\
\text { OR Point Estimate* (95\% CI) }\end{array}$ \\
\hline \multicolumn{3}{|l|}{ Demographics } \\
\hline Occupation: Unemployed/ retired vs. Working & $1.11(0.66-1.84)$ & $2.23(1.18-4.22)$ \\
\hline Occupation: Student vs. Working & $0.49(0.23-1.07)$ & $0.97(0.42-2.27)$ \\
\hline \multicolumn{3}{|l|}{ Severity of Injury } \\
\hline $\begin{array}{l}\text { Type of wheelchair: Manual wheelchair vs. No } \\
\text { wheelchair use }\end{array}$ & $1.95(1.26-3.01)$ & $1.73(1.00-2.99)$ \\
\hline $\begin{array}{l}\text { Type of wheelchair: Power assist wheelchair vs. } \\
\text { No wheelchair use }\end{array}$ & $0.83(0.17-4.01)$ & $0.63(0.07-5.51)$ \\
\hline $\begin{array}{l}\text { Type of wheelchair: Power wheelchair vs. No } \\
\text { wheelchair use }\end{array}$ & $1.83(0.43-1.59)$ & $2.04(1.11-3.72)$ \\
\hline \multicolumn{3}{|l|}{ Psychosocial Factors } \\
\hline Depression: No vs. Yes & $0.51(0.27-0.98)$ & $0.22(0.11-0.44)$ \\
\hline Primary caregiver: No one vs. Hired caregiver & $2.45(0.67-8.94)$ & $0.66(0.07-6.03)$ \\
\hline $\begin{array}{l}\text { Primary caregiver: Family/ significant other vs. } \\
\text { Hired caregiver }\end{array}$ & $2.24(1.14-4.41)$ & $1.49(0.63-2.55)$ \\
\hline Caregiver trained at rehab: No vs. Yes & $0.48(0.27-0.88)$ & $0.51(0.23-1.14)$ \\
\hline Primary payer: Medicare vs. Private insurance & $1.40(0.64-3.03)$ & $1.44(0.53-3.91)$ \\
\hline Primary payer: Medicaid vs. Private insurance & $1.20(0.75-1.91)$ & $1.42(0.79-2.58)$ \\
\hline $\begin{array}{l}\text { Primary payer: Worker's compensation vs. } \\
\text { Private insurance }\end{array}$ & $0.80(0.44-1.45)$ & $2.25(1.09-4.62)$ \\
\hline \multicolumn{3}{|l|}{ Clinical Information } \\
\hline FIM Motor at admission & $0.99(0.98-1.02)$ & $0.99(0.97-1.03)$ \\
\hline FIM Motor at discharge & $1.02(1.00-1.03)$ & $1.02(0.99-1.04)$ \\
\hline FIM Motor 1 year follow-up & $0.96(0.95-0.98)$ & $0.96(0.94-0.98)$ \\
\hline Physical therapy total hours & $0.99(0.98-0.99)$ & $0.98(0.96-0.99)$ \\
\hline
\end{tabular}

\section{Results}

Out of 1170 participants, 228 participants (19.4\%) were readmitted once and $120(10.2 \%)$ were readmitted multiple times within one year following discharge from IRF. As presented in Table 1, Chisquare analysis indicated that education $(\chi(16)=27.78, \mathrm{p}=0.03)$, occupation $(\chi(12)=38.94, \mathrm{p}<0.001)$, marital status $(\chi(12)=24.20, \mathrm{p}=0.02)$, AIS motor index score $(\chi(8)=33.50, \mathrm{p}<0.001)$, mechanical ventilation at $\operatorname{IRF}(\chi(6)=16.21, \mathrm{p}=0.13)$, mode of locomotion $(\chi(6)=22.55, \mathrm{p}<0.001)$, type of wheelchair $(\chi(12)=60.58, \mathrm{p}<0.001)$, traumatic etiology $(\chi(8)=27.56, \mathrm{p}<0.001)$, depression $(\chi(6)=31.58, \mathrm{p}<0.001)$, access to residence $(\chi(6)=14.22, \mathrm{p}=0.02)$, access to transportation $(\chi(6)=19.86, \mathrm{p}=0.01)$, primary caregiver $(\chi(10)=36.68, \mathrm{p}<0.001)$, caregiver training at rehab $(\chi(4)=21.23, \mathrm{p}<0.001)$, and community participation $(\chi(4)=14.91, \mathrm{p}=0.01)$ were different for between-group analysis.

In addition, ANOVA analysis indicated that FIM motor scores at admission $(\mathrm{F}(2,1167)=6.62, \mathrm{p}<0.001)$, discharge $(\mathrm{F}(2,1167)=28.80, \mathrm{p}<0.001)$, and one-year follow-up $(\mathrm{F}(2,1107)=45.27, \mathrm{p}<0.001)$, number of days from injury to initial IRF admission $(\mathrm{F}(2,1167)=15.16, \mathrm{p}<0.001)$, and total hours of physical therapy $(\mathrm{F}(2,1167)=4.33, \mathrm{p}=0.01)$, speech-language pathology $(\mathrm{F}(2,1167)=8.18, \mathrm{p}<0.001)$, therapeutic recreation $(\mathrm{F}(2,1167)=5.52, \mathrm{p}=0.01)$, and nursing $(\mathrm{F}(2,1167)=5.37, \mathrm{p}=0.01)$ during IRF stay were different for between-group analysis. Post-Hoc Bonferroni test revealed individuals with no readmissions had significantly higher FIM scores individuals with single $(\mathrm{p}=0.02$ [admission], $\mathrm{p}<0.001$ [discharge], $\mathrm{p}<0.001$ 
[1-year follow-up]) and multiple ( $\mathrm{p}=0.02$ [admission], $\mathrm{p}<0.001$ [discharge], $\mathrm{p}<0.001$ [1-year follow-up]) readmissions. Individuals with no readmissions also had fewer days from initial injury to IRF admission than individuals with single $(p<0.001)$ and multiple readmissions $(p<0.001)$. Individuals with no readmission had more hours of physical therapy $(\mathrm{p}=0.02)$ and speech-language pathology $(\mathrm{p}<0.001)$ than individuals with multiple readmissions. Individuals with no readmissions had more hours of therapeutic recreation than individuals with single $(\mathrm{p}=0.04)$ and multiple $(\mathrm{p}=0.03)$ readmissions. Lastly, individuals with no readmissions had fewer hours of nursing than individuals with multiple readmissions $(\mathrm{p}=0.01)$.

In addition to these findings, Table 2 shows significantly greater risk of single hospital readmission in individuals who: (1) had a primary caregiver who was not trained at the IRF compared to a caregiver who was trained at the IRF (OR=0.48 (0.27-0.88)), (2) had a family member or significant other as a primary caregiver compared to a hired caregiver $(\mathrm{OR}=2.24$ (1.14-4.41)), and (3) had Medicaid as a primary insurance payer compared to a private insurance payer $(\mathrm{OR}=1.20(0.75-1.91))$. Multiple hospital readmissions were more likely in individuals who: (1) were unemployed compared to working $(\mathrm{OR}=2.23$ (1.18-4.22)), and (2) had worker's compensation as a primary insurance payer compared to a private insurance payer $(\mathrm{OR}=2.25(1.09-4.62))$. Both single and multiple readmis sions are less likely in individuals who: (1) had no depression ( $\mathrm{OR}=0.51$ (0.27-0.98) [single], $\mathrm{OR}=0.22(0.11-0.44)$ [multiple])), (2) had higher FIM score at 1 year follow-up $(\mathrm{OR}=0.96(0.95-0.98)$ [single], OR=0.96 (0.94-0.98) [multiple])), (3) received more total hours of physical therapy during IRF stay (OR=0.99 (0.98-0.99) [single], OR=0.98 (0.96-0.99) [multiple])), and (4) used a manual or power wheelchair compared to those who walk $\mathrm{OR}=1.95$ (1.26-3.01) [single], OR=1.73 (1.00-2.99) [multiple])).

As displayed in Figure 1, of the 120 individuals with multiple readmissions, 41 (34.2\%) were readmitted at least once due to a genitourinary condition. Thirteen of $120(10.8 \%)$ were readmitted at least once due to a respiratory condition, and five of $120(4.2 \%)$ were readmitted at least once due to a skin condition. Out of 41 participants readmitted at least once for a genitourinary condition, approximately half (51.3\%) were readmitted more than once for a genitourinary related condition. Nine of the 13 participants $(69.3 \%)$ who had multiple readmissions were readmitted more than once for a respiratory related condition. No participants were readmitted more than once due to a skin related condition.

\section{Discussion}

To our knowledge, this is the first study using longitudinal data to confirm the differences in clinical and psychosocial characteristics in individuals with SCI with multiple hospital readmissions. Hospital readmission rates within 30 days of discharge are higher in SCI $(17.4 \%)$ than other conditions, including stroke $(12.7 \%)$ and brain dys function $(16.4 \%) .^{2}$ We stratified readmis sions to specifically examine factors related to multiple readmissions separately from single readmissions. Furthermore, we analyzed rich casemix variables not present in other studies of multiple readmissions such as information on occupation, caregivers, and type of wheelchair.

\section{Function and Injury Level}

Consistent with previous research, we found that lower FIM motor score was significantly associated with increased risk of hospital readmission. $13,14,16,17$ Our findings suggest that individuals who primarily use a manual or power wheelchair (proxy measure of higher level of impairment) are more likely to be readmitted multiple times than individuals who do not use a wheelchair. This is likely due to the fact that the individuals who do not use a wheelchair are typically ambulatory, and therefore higher functioning. 
Our study further deconstructs discipline specific information on rehabilitation services and the likelihood of hospital readmission. Total hours of treatment across all disciplines while at IRF was approximately the same in individuals with no readmissions, one readmission, and multiple readmissions (189.7, 189.4, 185.3 hours respectively). However, individuals receiving a lower amount of physical therapy have higher odds of multiple readmissions, which is consistent with previous findings that fewer hours of physical therapy is associated with increased odds of readmis sion. ${ }^{13}$ On average, individuals with multiple readmissions received less hours of physical therapy, but more hours of nursing, psychology, and social work than the group with no readmissions. This may indicate that other prevailing needs of rehabilitation services were addressed in place of physical therapy, or that the participants were unable to participate in the same amount of therapy.

\section{Psychosocial Factors and Psychological Well-Being}

Our findings clearly establish an association between social determinants of health and long-term health outcomes over the year. Decreased caregiver support is significantly associated with increased risk for hospital readmission. Individuals with a family member as a primary caregiver opposed to a hired caregiver are more likely to be readmitted once. However, evidence supports that quality of care provided by caregivers who are family members tends to be equal to or exceed quality of care provided by hired caregivers. ${ }^{27}$ There was no significant difference between caregiver types in the group of multiple readmis sions. Individuals whose caregiver was not trained at the IRF had higher odds of being readmitted once, which reinforces the findings of previous research that caregiver training has positive results. ${ }^{27}$ Therefore, ensuring caregivers are skilled is a vital component to preventing readmissions. Caregivers are vital for individuals with SCI to transition from inpatient rehabilitation to independence at home to facilitate community reintegration. ${ }^{28}$ It may be inferred that individuals who do not have a caregiver are either sufficiently functionally independent and thus do not require caregiver assistance, or they would benefit from caregiver assistance but lack access to resources that would provide a caregiver. Participant's lack of caregiver for each of these reasons would expectedly result in different risks of readmission. However, since the reason for lack of caregiver is not divulged, we are unable to separately evaluate the effect of each reason for lack of caregiver. Discharge planning should account for ensuring appropriate caregiver services, however additional follow-up with patients from social workers along with other members of the care team may assist to prevent consequential readmissions if caregiver support changes after discharge.

Unemployment was linked to multiple readmissions, as well as having worker's compensation as a primary insurance payer. Financial hardship may influence readmission for individuals who are unable to afford preventative care measures not covered by insurance, or who lack resources to seek ambulatory care for minor issues. If paying a copay is a deterrent to a physician visit for a persistent cough, perhaps patients may be susceptible to developing more serious respiratory infections requiring hospitalization. In similar circumstances, readmissions may be avoided by proper management and timely intervention of arising issues.

Individuals with depression were more likely to be readmitted once and multiple times. DeJong et al.13 found that depressive symptoms were more prevalent in individuals who were readmitted, but questioned whether depression increases risk of readmission or if readmission increases risk for depression. Depression is common in individuals following SCI, and often accompanied by behavioral problems, substance abuse, and other psychological disorders that necessitate educational interventions for patient management.29-31 The impact of depression on self-care abilities and cognition may contribute to the relationship between depression and recurrence of readmissions. Furthermore, memory and learning deficits due to depression may hinder retention of educational interventions to prevent health related complications that lead to readmissions. 


\section{Genitourinary, Respiratory, and Skin Conditions}

This study provided information on the impact of three common medical complications in individuals with SCI. DeJong et al. ${ }^{13}$ examined multiple readmissions but was unable to determine rates of readmission for these conditions due to missing data in $40.2 \%$ of the sample used. Our criteria excluded individuals with unknown causes of readmission, enabling our analysis of these conditions in our cohort. Genitourinary, respiratory, and skin conditions are leading medical diagnoses as sociated with readmis sion among individuals with SCI. ${ }^{11,14,20}$ These conditions are common and often recurrent, and readmissions for these diagnoses are considered preventable, which means community-based preventive management is crucial to avert ongoing repercussions. Timely assessment and effective preventative measures can reduce the risk of multiple readmissions. Our findings did not support that skin conditions were associated with multiple readmissions. We postulate that this is due to successful prophylaxis and outpatient wound care management, resulting in prevention of complications that require hos pitalization. Preventative measures for genitourinary and respiratory complications may be less effective due to the aforementioned psychosocial factors, resulting in recurrent hospitalizations. It is also possible that these complications are inclined to be more complex and challenging to manage than pressure injuries, which would explain the higher frequency of hospital readmission.

\section{Implications for Future Research}

Socioeconomic and psychosocial patient factors that are not captured by Medicare claims and adminis trative intake should be considered in designing post-acute discharge planning and intervention to identify issues with patient compliance, cognitive deficits, and comorbidities that may influence readmission rates. Methods to prevent readmissions have been evolving, however we presume that preventing multiple readmissions requires a different approach than what has been proposed for all-cause readmissions in prior literature. ${ }^{13}$ Future studies should investigate how to address social determinants of health to prevent multiple readmissions, including the examination of educational strategies and caregiver training. Collaboration between health care systems to collect longitudinal information regarding readmissions and psychosocial factors, caregiver support, utilization of outpatient services and homecare services, and patient compliance would improve critical understanding. This insight could assist in the development of an all-encompassing approach that recognizes risk factors in individuals to better determine who is susceptible to multiple readmissions, and focus on risk factor modification. We hope that with additional perspective into the cause of multiple readmissions among individuals with SCI, efficacy of preventative methods will be maximized, thus reducing the economic burden on patients and health care systems.

\section{Limitations}

The primary limitation of this study was the small sample size of the multiple readmissions group, and some factors may have been underrepresented due to missing data. Nevertheless, this dataset has provided comprehensive patient information. Another limitation of this study was self-reported one-year follow-up data. Information regarding readmissions was collected through patient surveys, so inadequate patient recall may have resulted in underestimation of readmission rates. Lastly, we were unable to perform analyses based on clustering within the SCI centers and were unable to compare geographical impact due to the lack of this information in the publicly available dataset. Future studies are recommended to examine the impact of regional availability of post-acute rehab services and social determinants of health on patient outcomes. 


\section{Conclusions}

This study found that lower utilization of rehabilitation services, lower functional status, unemployment, and depression were as sociated with increased incidence of multiple readmissions within one year postdischarge from IRF. In order to reduce recurrent readmissions in individuals with SCI, further research is necessary to improve risk factor modification. In order to modify risk factors effectively, efforts should focus on implementation of prevention strategies focused on coordination of follow-up care, caregiver training and education on post-discharge instructions, and ensuring appropriate transition of care.

\section{Disclosures and Conflicts of Interest}

The authors affirm that we do not have any involvement with any commercial organization that has a direct financial interest in any matter included in this manuscript.

\section{Funding Sources}

Drs. Canori and Hiremath's work on this project was supported by intramural research funds from the College of Public Health, Temple University. Dr. Kumar's work on this article was supported by the College of Health \& Human Services, Northern Arizona University, and the National Institutes of Health (R03AG060345, R03HD096372, and U54MD012388).

\section{References}

1. Zuckerman RB, Sheingold SH, Orav EJ, Ruhter J, Epstein AM. Readmissions, observation, and the Hospital Readmissions Reduction Program. The New England journal of medicine. 2016;374(16):1543-51. doi: 10.1056/NEJMsa1513024. PubMed PMID: 26910198.

2. Ottenbacher KJ, Karmarkar A, Graham JE, Kuo Y-F, Deutsch A, Reistetter TA, et al. Thirty-Day Hospital Readmission Following Discharge From Postacute Rehabilitation in Fee-for-Service Medicare Patients. Jama. 2014;311(6):604-14. doi: 10.1001/jama.2014.8.

3. IRF quality reporting measures information [November 29, 2018]. Available from: https:/ / www.cms.gov/Medicare/Quality-Initiatives-Patient-Assessment-Instruments/IRF-QualityReporting/IRF-Quality-Reporting-Program-Measures-Information-.html.

4. Zuckerman RB, Joynt Maddox KE, Sheingold SH, Chen LM, Epstein AM. Effect of a hospital-wide measure on the readmissions reduction program. The New England journal of medicine. 2017;377(16):1551-8. doi: 10.1056/NEJMsa1701791. PubMed PMID: 29045205.

5. Prospective payment systems for inpatient hospital services 2018 [November 1, 2018]. Available from: https://www.govinfo.gov/app/details/CFR-2011-title42-vol2/CFR-2011-title42-vol2part412.

6. Jencks SF, Williams MV, Coleman EA. Rehospitalizations among patients in the Medicare fee-forservice program. The New England journal of medicine. 2009;360(14):1418-28. doi: 10.1056/ NEJMsa0803563. PubMed PMID: 19339721.

7. Readmissions Reduction Program (Medicare Payment Advisory Commission W, DC) Available at:. http://www.medpac.gov/docs/default-source/reports/jun18 ch1 medpacreport sec.pdf. June 2018.

8. Goldfield NI, McCullough EC, Hughes JS, Tang AM, Eastman B, Rawlins LK, et al. Identifying potentially preventable readmissions. Health care financing review. 2008;30(1):75-91. PubMed PMID: 19040175; PubMed Central PMCID: PMC4195042.

9. Development of potentially preventable readmission measures for post-acute care October 2015 [January 9, 2019]. Available from: https://www.cms.gov/Medicare/Quality-Initiatives-Patient- 
Assessment-Instruments/MMS/Downloads/Draft-Measure-Specifications-for-PotentiallyPreventable-Hospital-Readmission-Measures-for-PAC-.pdf.

10. Greenwald JL, Jack BW. Preventing the preventable: reducing rehospitalizations through coordinated, patient-centered discharge processes. Professional case management. 2009;14(3):13540; quiz 41-2. doi: 10.1097/ NCM.0b013e318198d4e1. PubMed PMID: 19474639; PubMed Central PMCID: PMC2720804.

11. Dryden DM, Saunders LD, Rowe BH, May LA, Yiannakoulias N, Svenson LW, et al. Utilization of health services following spinal cord injury: a 6-year follow-up study. Spinal cord. 2004;42(9):513-25. doi: 10.1038/sj.sc.3101629. PubMed PMID: 15249928.

12. Skelton F, Hoffman JM, Reyes M, Burns SP. Examining health-care utilization in the first year following spinal cord injury. The journal of spinal cord medicine. 2015;38(6):690-5. doi: 10.1179/2045772314Y.0000000269. PubMed PMID: 25299152; PubMed Central PMCID: PMC4725802.

13. DeJong G, Tian W, Hsieh CH, Junn C, Karam C, Ballard PH, et al. Rehospitalization in the first year of traumatic spinal cord injury after discharge from medical rehabilitation. Archives of physical medicine and rehabilitation. 2013;94(4 Suppl):S87-97. doi: 10.1016/j.apmr.2012.10.037. PubMed PMID: 23527776.

14. Cardenas DD, Hoffman JM, Kirshblum S, McKinley W. Etiology and incidence of rehospitalization after traumatic spinal cord injury: a multicenter analysis. Archives of physical medicine and rehabilitation. 2004;85(11):1757-63. PubMed PMID: 15520970.

15. Jaglal SB, Munce SE, Guilcher SJ, Couris CM, Fung K, Craven BC, et al. Health system factors associated with rehospitalizations after traumatic spinal cord injury: a population-based study. Spinal cord. 2009;47(8):604-9. doi: 10.1038/sc.2009.9. PubMed PMID: 19274059.

16. Mahmoudi E, Meade MA, Forchheimer MB, Fyffe DC, Krause JS, Tate D. Longitudinal analysis of hospitalization after spinal cord injury: variation based on race and ethnicity. Archives of physical medicine and rehabilitation. 2014;95(11):2158-66. doi: 10.1016/j.apmr.2014.07.399. PubMed PMID: 25094001.

17. Pretz CR, Graham JE, Middleton A, Karmarkar AM, Ottenbacher KJ. Longitudinal investigation of rehospitalization patterns in spinal cord injury and traumatic brain injury among Medicare beneficiaries. Archives of physical medicine and rehabilitation. 2017;98(5):997-1003. doi: 10.1016/j.apmr.2016.12.012. PubMed PMID: 28115070; PubMed Central PMCID: PMC5403607.

18. Yarbrough CK, Gamble PG, Burhan Janjua M, Tang M, Ghenbot R, Zhang AJ, et al. Readmission after spinal cord injury: analysis of an institutional cohort of 795 patients. Journal of neurosurgical sciences. 2018;62(3):265-70. doi: 10.23736/S0390-5616.16.03664-X. PubMed PMID: 27152452.

19. Stillman MD, Frost KL, Smalley C, Bertocci G, Williams S. Health care utilization and barriers experienced by individuals with spinal cord injury. Archives of physical medicine and rehabilitation. 2014;95(6):1114-26. doi: 10.1016/j.apmr.2014.02.005. PubMed PMID: 24565745.

20. Middleton JW, Lim K, Taylor L, Soden R, Rutkowski S. Patterns of morbidity and rehospitalisation following spinal cord injury. Spinal cord. 2004;42(6):359-67. doi: 10.1038/sj.sc.3101601. PubMed PMID: 15007376.

21. Whiteneck G, Gassaway J, Dijkers M, Jha A. New approach to study the contents and outcomes of spinal cord injury rehabilitation: the SCIRehab Project. The journal of spinal cord medicine. 2009;32(3):251-9. PubMed PMID: 19810627; PubMed Central PMCID: PMC2718827.

22. Whiteneck G, Gassaway J, Dijkers M, Backus D, Charlifue S, Chen D, et al. The SCIRehab project. treatment time spent in SCI rehabilitation. Inpatient treatment time across disciplines in spinal cord injury rehabilitation. The journal of spinal cord medicine. 2011;34(2):133-48. doi: 10.1179/107902611X12971826988011. PubMed PMID: 21675353; PubMed Central PMCID: PMC3066504. 
23. Jesus TS, Hoenig H. Postacute rehabilitation quality of care: toward a shared conceptual framework. Archives of physical medicine and rehabilitation. 2015;96(5):960-9. doi: 10.1016/j.apmr.2014.12.007. PubMed PMID: 25542676.

24. Qu H, Shewchuk RM, Chen YY, Richards JS. Evaluating the quality of acute rehabilitation care for patients with spinal cord injury: an extended Donabedian model. Quality management in health care. 2010;19(1):47-61. doi: 10.1097/QMH.0b013e3181ccbc2a. PubMed PMID: 20042933.

25. Ravaud JF, Delcey M, Yelnik A. Construct validity of the functional independence measure (FIM): questioning the unidimensionality of the scale and the "value" of FIM scores. Scandinavian journal of rehabilitation medicine. 1999;31(1):31-41. PubMed PMID: 10230001.

26. Kroenke K, Spitzer RL, Williams JB. The PHQ-9: validity of a brief depression severity measure. Journal of general internal medicine. 2001;16(9):606-13. Epub 2001/09/15. doi: 10.1046/j.15251497.2001.016009606.x. PubMed PMID: 11556941; PubMed Central PMCID: PMCPMC1495268.

27. Smith EM, Boucher N, Miller WC. Caregiving services in spinal cord injury: a systematic review of the literature. Spinal cord. 2016;54:562. doi: 10.1038/sc.2016.8.

28. Moreno A, Zidarov D, Raju C, Boruff J, Ahmed S. Integrating the perspectives of individuals with spinal cord injuries, their family caregivers and healthcare professionals from the time of rehabilitation admission to community reintegration: protocol for a scoping study on SCI needs. BMJ open. 2017;7(8):e014331. Epub 2017/08/07. doi: 10.1136/bmjopen-2016-014331. PubMed PMID: 28780539; PubMed Central PMCID: PMCPMC5724190.

29. Elliott TR, Frank RG. Depression following spinal cord injury. Archives of physical medicine and rehabilitation. 1996;77(8):816-23. PubMed PMID: 8702378.

30. Elliott TR. Studying depression following spinal cord injury: Evidence, policy and practice. The journal of spinal cord medicine. 2015;38(5):584-6. doi: 10.1179/2045772315Y.0000000046. PubMed PMID: 26181901; PubMed Central PMCID: PMC4535799.

31. Dryden DM, Saunders LD, Rowe BH, May LA, Yiannakoulias N, Svenson LW, et al. Depression following traumatic spinal cord injury. Neuroepidemiology. 2005;25(2):55-61. doi: 10.1159/000086284. PubMed PMID: 15947491.

\section{Statement of Contributions}

AC: Study design, conducting the study, data analyses, manuscript preparation AK: Study design, data analyses, manuscript review and editing

SVH: Study design, conducting the study, data analyses, manuscript preparation 